\title{
Therapeutic efficacy of artesunate in the treatment of uncomplicated Plasmodium falciparum malaria and anti-malarial, drug-resistance marker polymorphisms in populations near the China-Myanmar border
}

Fang Huang ${ }^{1}$, Linhua Tang ${ }^{1 *}$, Henglin Yang ${ }^{2}$, Shuisen Zhou' ${ }^{1}$ Xiaodong Sun ${ }^{2}$ and Hui Liu ${ }^{2}$

\begin{abstract}
Background: The aim of this study was to evaluate the clinical outcome after seven-day artesunate monotherapy for uncomplicated Plasmodium falciparum malaria in Yingjiang County along the China-Myanmar border and investigate genetic polymorphisms in the P. falciparum chloroquine-resistance transporter ( $p f c r t)$, multidrug resistance 1 (pfmdr1), dihydrofolate reductase (pfdhfr), dihydropteroate synthase (pfdhps) and ATPase (pfatp6) genes.

Methods: Patients $\geq$ one year of age with fever (axillary temperature $\geq 37.5^{\circ} \mathrm{C}$ ) or history of fever and $P$. falciparum mono-infection were included. Patients received anti-malarial treatment with artesunate (total dose of $16 \mathrm{mg} / \mathrm{kg}$ over seven days) by directly observed therapy. After a 28-day follow-up, treatment efficacy and effectiveness were assessed based on clinical and parasitological outcomes. Treatment failure was defined as recrudescence of the original parasite and distinguished with new infection confirmed by PCR. Analysis of gene mutation and amplification were performed by nested polymerase chain reaction.

Results: Sixty-five patients were enrolled; 10 withdrew from the study, and six were lost to follow-up. All but two patients demonstrated adequate clinical and parasitological response; 12 had detectable parasitaemia on day 3 . These two patients were confirmed to be new infection by PCR. The efficacy of artesunate was $95.9 \%$. The pfcrt mutation in codon 76 was found in all isolates (100\%), and mutations in codons 71 and 72 were found in $4.8 \%$ of parasite isolates. No mutation of pfmdr 1 (codons 86 or 1246) was found. Among all samples, $5.1 \%$ were wild type for pfdhfr, whereas the other samples had mutations in four codons (51,59, 108 and 164), and mutations in pfdhps (codons 436, 437, 540 and 581) were found in all isolates. No samples had mutations in pfatp6 codons 623 or 769, but two new mutations (N683K and R756K) were found in 4.6\% and 9.2\% of parasite isolates, respectively.

Conclusion: Plasmodium falciparum infection was associated with slow parasite clearance and suspected artemisinin resistance at the China-Myanmar border area. The prevalence of pfcrt $76 \mathrm{~T}$ and markers for SP resistance are still high. It should be strengthened further on parasite clearance time or clearance half life to confirm the resistance status, and molecular epidemiology should provide complementary information to assess the appropriateness of current policies based on artemisinin derivatives.
\end{abstract}

Keywords: Molecular markers, Artesunate, Plasmodium falciparum

\footnotetext{
* Correspondence: LinhuaTang-ipdtlh@sh163.net

${ }^{1}$ National Institute of Parasitic Diseases, Chinese Centre for Disease Control and Prevention; WHO Collaborating Centre for Malaria, Schistosomiasis and Filariasis; Key Laboratory of Parasite and Vector Biology, Ministry of Health, Shanghai 200025, PR China

Full list of author information is available at the end of the article
} 


\section{Background}

Malaria is a severe infectious disease, and drug resistance to anti-malarial drugs is a major public health problem worldwide [1]. In the last five years, there has been increasing concern about the emergence of resistance to artemisinin anti-malarials in Southeast Asia [2]. Several publications, including WHO reports, have provided evidence for the presence of Plasmodium falciparum tolerance/resistance to artesunate in populations on the Cambodia-Thailand border $[3,4]$.

Falciparum malaria is now found in only two provinces in China: Yunnan province, which borders Myanmar, Laos and Vietnam, and Hainan province. Malaria control measures have been actively implemented in this area for more than 30 years with considerable success, and there are no local falciparum malaria cases in Hainan [5]. However, the malaria situation at the ChinaMyanmar border remains serious.

China was the first country to use artemisinins, but their wide-scale use in China began only in the early 1990s. The standard treatment regimen was $12 \mathrm{mg} / \mathrm{kg}$ artesunate or artemether over five days, which continued to be widely used until 2007. Since 2001, the WHO has recommended the use of artemisinin-based combination therapy in all areas where $P$. falciparum is resistant to other anti-malarial medicines to optimize therapeutic effectiveness and delay the emergence of resistance. In 2006, the WHO advocated a complete ban on artemisinin monotherapy for uncomplicated malaria [6]. The national drug policy of China was updated in 2009, and since then, the first-line drugs used to treat falciparum malaria has been artemisinin-based combination therapy (ACT), which includes dihydroartemisinin-piperaquine, artesunate-amodiaquine, artimisinin-naphthoquine phosphate and artemisinin-piperaquine [7].

Until now, ACT has been the only effective treatment for falciparum malaria. At a time when ACT is being rolled out in endemic malaria areas all over the world and is beginning to have an effect on morbidity and mortality in tropical Africa, the development and spread of ACT resistance would have grave consequences for global malarial control. Because of these concerns, national malaria programmes conduct systematic periodic in vivo malaria therapeutic efficacy studies in sentinel sites to monitor P. falciparum and Plasmodium vivax sensitivity to first-line, anti-malarial treatments.

Several genetic polymorphisms that can provide reliable data about the prevalence of drug resistance have been described in $P$. falciparum and $P$. vivax. The most relevant polymorphisms are presented below. The $76 \mathrm{~T}$ allele in the chloroquine-resistance transporter gene ( $p f c r t)$ is predictive of chloroquine and amodiaquine treatment failure [8-10]. The $86 \mathrm{Y}$ allele of the multidrug resistance gene 1 (pfmdr1) has been linked to chloroquine and amodiaquine resistance, and increased chloroquine inhibitory concentrations in P. falciparum have been linked with pfcrt $76 \mathrm{~T}$ [11]. The triple dihydrofolate reductase (pfdhfr) haplotype N51I/C59R/S108N has been associated with sulphadoxine-pyrimethamine (SP) treatment failure, and the addition of the dihydropteroate synthase (pfdhps) SNPs G437A and K540E produces highly resistant P. falciparum [12-16]. The sarco/ endoplasmic reticulum $\mathrm{Ca}^{2+}$-ATPase ortholog of $P$. falciparum (pfatp6) was suggested to be involved in the mechanism of action and resistance of the parasite to artemisinins [17].

The aim of this study was to evaluate clinical outcome after seven-day artesunate monotherapy for uncomplicated P. falciparum malaria in Yingjiang County along the China-Myanmar border and to investigate the prevalence of genetic polymorphisms in pfcrt, pfmdr1, pfdhfr, pfdhps and pfatp6.

\section{Methods}

\section{Study sites and design}

The study was a one-arm prospective evaluation of clinical and parasitological response to directly observe treatment for uncomplicated malaria. The study was conducted in Yingjiang County, which is near Lazan City in Myanmar.

\section{Recruitment of patients}

Patients over six months of age with fever (axillary temperature $\geq 37.5^{\circ} \mathrm{C}$ ) or history of fever in the previous 48 hours were included. The included patients had mono-infection with $P$. falciparum, parasitaemia levels between 250 and 100,000 asexual parasites/ $\mu$ l, no history of anti-malarial use in the previous 14 days and no signs of severe malaria or danger signs.

\section{Treatment of patients and follow-up}

After written informed consent was obtained, a detailed medical history, clinical examination and both thick and thin blood films were performed. Artesunate was administered at a total dose of $16 \mathrm{mg} / \mathrm{kg}$ bw over seven days (first day: $4 \mathrm{mg} / \mathrm{kg}$ bw; second to seventh days: $2 \mathrm{mg} / \mathrm{kg}$ bw/day). Direct observed therapy (DOTS) was given for seven days by the health worker. Tablets of artesunate were obtained from the WHO (manufactured by Guilin Pharmaceutical Co., Ltd., Guilin, China). In case of vomiting within 30 minutes, the dose was repeated; if vomiting occurred within 30 minutes after the repeated dose, the patients were excluded from the study and referred for treatment at the healthcare facility.

Patients were followed up on days $1,2,3,4,5,6,7,14$, 21 and 28 for clinical and laboratory tests, which included axillary temperature measurement and thick blood smear preparation. If a patient could not be located 
on the day of the visit, the study staff attempted to locate the patient at his or her home. Patients who were not located within one day of the expected visit were considered lost to follow-up and excluded from the study.

Sample size was calculated based on a predicted treatment failure rate of $15 \%$ because the treatment failure rate of artesunate in the area was unknown. At a confidence level of $95 \%$ and a precision of approximately $5 \%$, a minimum of 50 patients were required for the drug to be tested. At least 60 patients should be included to allow up to $20 \%$ loss to follow-up and withdrawals during the 28-day follow-up period.

Thick blood smears obtained on every visit day were stained with $10 \%$ Giemsa for 10 minutes and examined at $1,000 \times$ magnification by a trained microscopist to confirm the species and parasite density. The parasite density was determined by counting the number of asexual forms and gametocytes in 200 leukocytes. Parasitaemia was estimated by assuming 6,000 leukocytes/ $\mu$ l. A second microscopist blinded to the first result reexamined the thick blood smear, and in case of discrepancies greater than $50 \%$, the smear was read by a third microscopist. The geometric mean of the two closest results was used as the parasitaemia value for each reading. When the number of asexual parasites was less than 10 per 200 white blood cells in follow-up smears, counting was performed in at least 500 white blood cells (ie, to completion of the field in which the 500th white blood cell was counted). A blood slide was considered to be negative when examination of 1,000 white blood cells revealed no asexual parasites. The presence of gametocytes on an enrolment or follow-up slide was noted, but this information did not contribute to the basic evaluation.

Genotype analysis was conducted to differentiate recrudescence (same parasite strain) from newly acquired infection (different parasite strain). This analysis is based on the extensive genetic diversity among the malaria parasite genes $m s p 1, m s p 2$ and glurp [18]. The genotypic profiles of pre- and post-parasite strains are compared.

\section{Outcome measures}

Treatment efficacy and effectiveness were evaluated among observed patients based on clinical and parasitologic outcomes and study endpoints in accordance with WHO guidelines for in vivo efficacy monitoring [18]. The outcomes were classified as early treatment failure (ETF), late clinical failure (LCF), late parasitological failure (LPF) and adequate clinical and parasitological response (ACPR).

\section{Ethical considerations}

The study was reviewed and approved by the ethical committee of the Chinese Centre for Disease Control and Prevention (China CDC). This study was also registered at the website [19] under the number ACTRN12610001008011.

\section{Molecular marker analysis}

DNA was extracted from dried blood samples on filter papers using a QIAGEN mini kit and stored at $-20^{\circ} \mathrm{C}$ until future use. Nested PCR $[8,14,19,20]$ was used to amplify fragments of $p f c r t, p f m d r 1, p f d h f r, p f d h p s$ and pfatp6. Sequencing reactions were carried out using an ABI PRISM BigDye Terminator v3.1 Cycle Sequencing kit (Applied Biosystems, CA, USA) as specified by the manufacturer. The sequences of the amplicons were aligned with data published in the NCBI database by BLAST analysis.

\section{Results}

Study site

All of the patients were observed at the Rose Clinic in Lazan City, a small town on the Myanmar side of the border located $24^{\circ} 47^{\prime} 21.22^{\prime \prime} \mathrm{N}$ and $97^{\circ} 33^{\prime} 21.05^{\prime \prime} \mathrm{E}$ (Figure 1). The Rose Clinic is close to the town of Nabang in Yingjiang County, China, and the two towns are separated by the Lazan stream. The town of Myitkina is located approximately $78 \mathrm{~km}$ north of Lazan City.

\section{Patient characteristics}

A total of 1,095 febrile patients were screened for malaria by blood smear (microscopic examination). A total of 302 malaria patients were found, including 153 cases of $P$. vivax, 65 cases of $P$. falciparum malaria, four cases of Plasmodium malariae and 11 cases of mixed infection (Figure 2). All of the P. falciparum patients met the inclusion criteria.

Of the P. falciparum patients, 17 were female (26.2\%) and 48 were male $(73.8 \%)$. The age distribution was $3.0 \%$ under five years, $15.5 \%$ between five and 15 years and $81.5 \%>15$ years. There was a mild predominance of young adult males. All of the patients had a fever in the 48 hours preceding presentation to the clinic, and $73.8 \%$ $(48 / 65)$ exhibited an axillary temperature $>37.5^{\circ} \mathrm{C}$. The geometric mean parasite density was 69,535 parasites/ $\mu \mathrm{l}$. The probability of the patients with parasite on day 3 was correlated with the parasite densities on day 0 , that is, the more parasite densities the more probability of positive on days 3 Table 1.

The patients' ethnicity was also noted: $84 \%$ belonged to the Jingpo ethnic group, which is dominant on both sides of the border in this area; 13\% were Bamar (Myanmar majority group); and the remaining $3 \%$ belonged to the local Lisu ethnic group. Among the $7 \%$ who were Chinese citizens, most were Han, and a few belonged to the Jingpo group. 


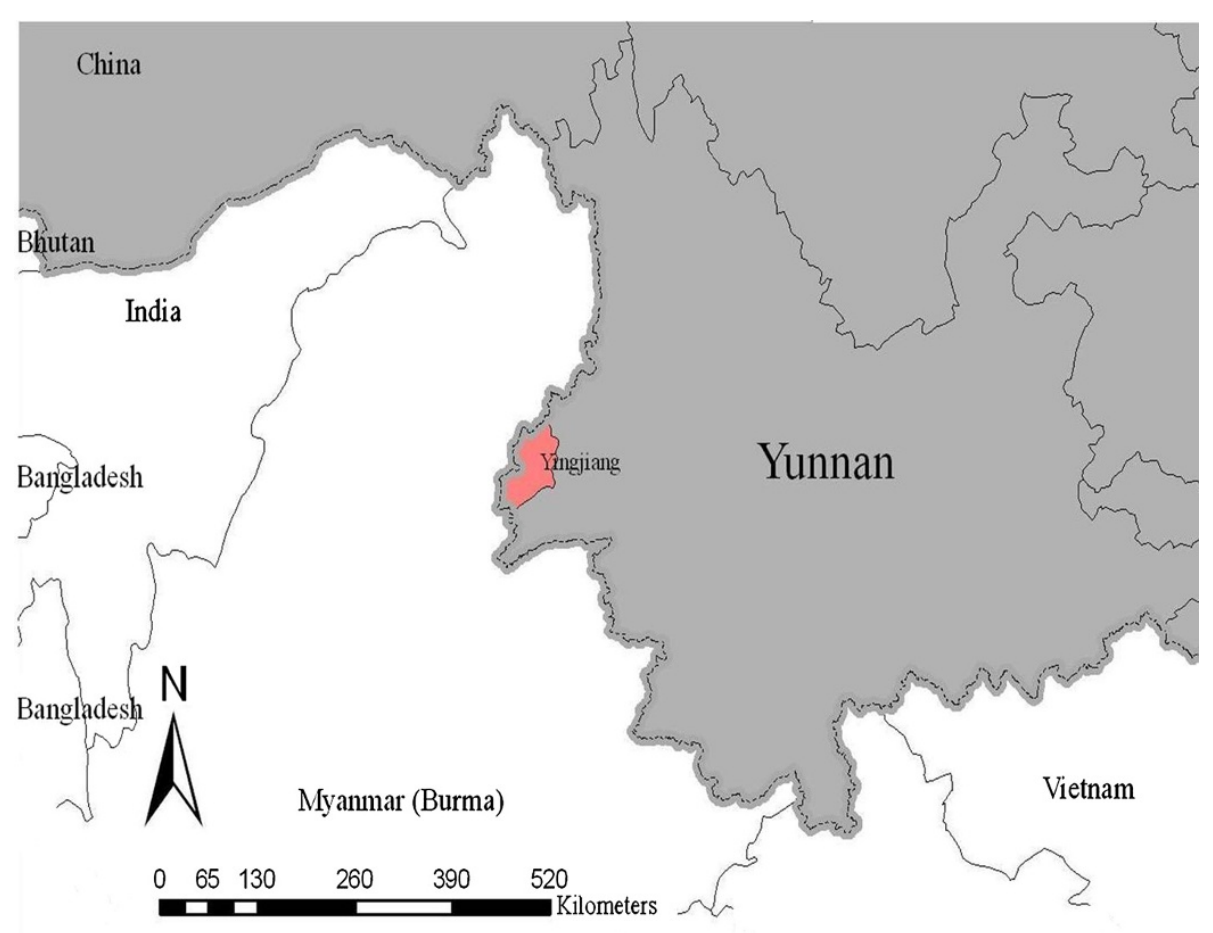

Figure 1 The location of Yingjiang County relative to neighbouring countries.

\section{Clinical outcomes}

Sixty-five patients were enrolled and received directly observed anti-malarial treatment with artesunate (total dose of $16 \mathrm{mg} / \mathrm{kg}$ over seven days); 10 withdrew from the study, and six were lost to follow-up. The efficacy of artesunate monotherapy was $95.9 \%$.

All but two patients in the observed group demonstrated adequate clinical and parasitological response. The two patients with treatment failure were confirmed to have new infection by PCR. The allelic variants of msp1, msp 2 and glurp were genotyped and the results of the pre- and post-parasite strains of the two patients were shown in Table 2.

Twelve patients had detectable parasitaemia on day 3 . The geometric mean parasite density on Day 0 in the cases that were positive on day 3 was 89,519 asexual parasites per $\mu \mathrm{l}$, indicating a strong association between day 3 positivity and high initial parasite density, as observed in other studies. The lowest day 0 parasitaemia associated with D3 positivity was 33,652 parasites/ $\mu$ l.

The average body temperature and parasite density of the patients decreased dramatically on days $0,1,2,3,7,14,21$ and 28 after seven days of artesunate treatment (Figure 3 ).

\section{Molecular markers for drug resistance Pfcrt and pfmdr1}

A total of 63 samples were successfully amplified at positions 71, 72 and 76. Mutation in pfcrt codon 76 was found in all of the isolates (100\%), and mutations in codons 71 and 72 were found in $4.8 \%$ of parasite isolates. No mutation in pfmdr1 (codons 86 or 1246) was found.

\section{Pfdhfr and pfdhps}

The pfdhfr genes from 63 samples were successfully amplified and compared with the wild-type sequence. The frequencies of N51I, C59R, and I164L are shown in Figure 4. The most prevalent mutations were C59R and S108N, which were present in $92.1 \%$ and $90.6 \%$ of patients, respectively (Table 3 ). The mixed infections including quadruple (38.0\%), triple (51.7) and double mutants $(10.3 \%)$ were described in Table 3.

For $p f d h p s$, successful amplification was achieved in 59 samples, and mutations in codons 436, 437, 540 and 581 were found in all of the isolates (100\%) including single, double, triple or quadruple mutants. The A437G and K540E mutants were most prevalent in pfdhps. Double mutants in pfdhps were found in $57.6 \%$ of samples and included 436/437, 436/540, 437/540, and 437/581 (Table 3). Except for one sample, all of the samples with the K540E mutation also had the A437G mutation.

\section{Pfatp6}

No mutation was found in pfatp6 codons 623 or 769 , but two new mutations (N683K and R756K) were found in $4.6 \%$ and $9.2 \%$ of parasite isolates, respectively. 


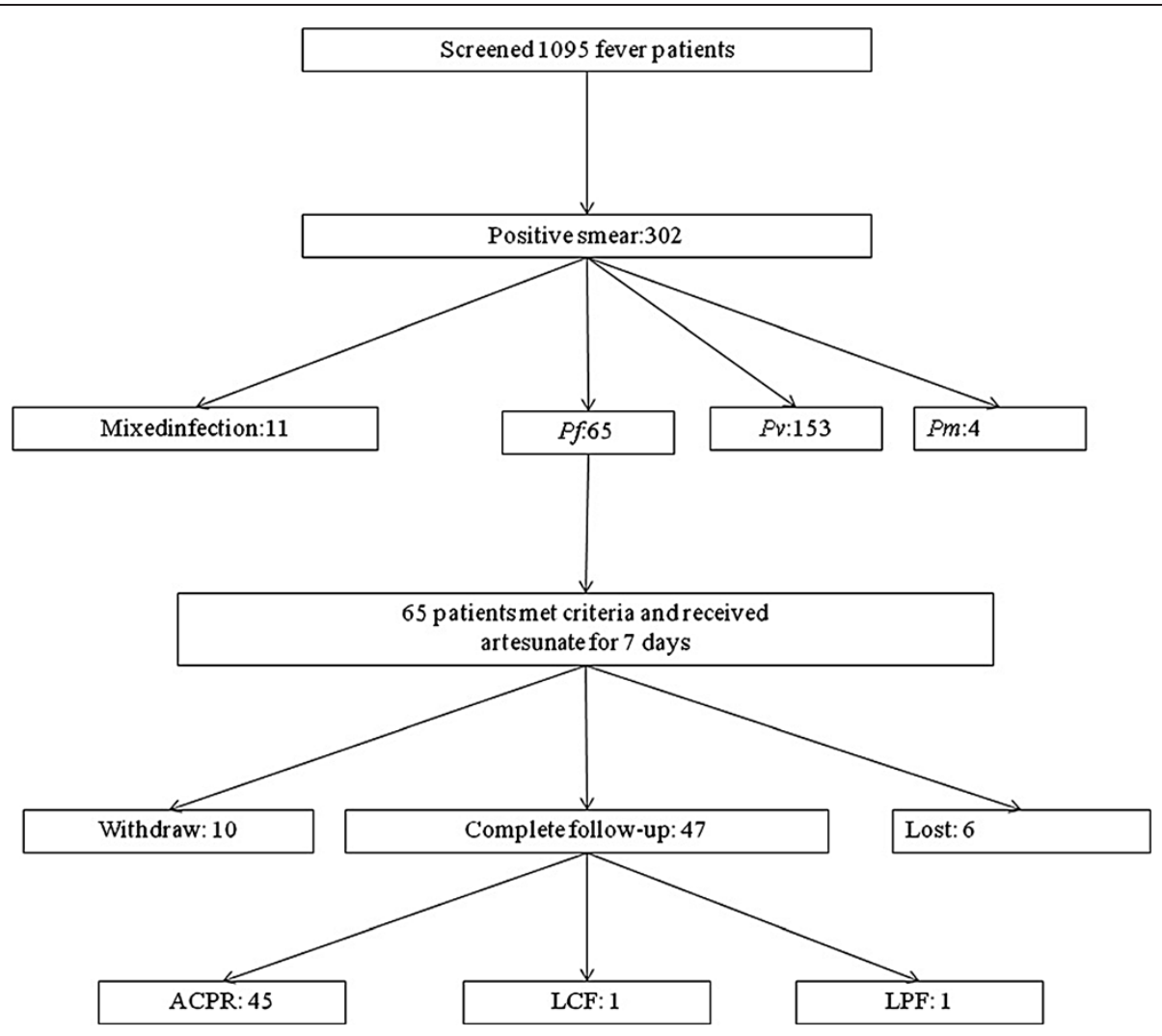

Figure 2 Patient recruitment and clinical outcomes.

\section{Discussion}

Artemisinin-based combination therapy is recommended to slow the emergence and spread of drug resistance. This effect is achieved by the use of anti-malarial drugs with different mechanisms of action and the use of a fast-acting highly effective anti-malarial drug, such as DHA, in combination with a long-lasting anti-malarial drug, such as piperaquine. China was the first country to use artemisinin, and this drug was used for malaria treatment in China for almost 20 years (from the 1990s to 2007). In recent years, some reports confirmed artemisinin resistance in Southeast Asia, especially near

Table 1 Characteristics of patients with Plasmodium falciparum

\begin{tabular}{lll}
\hline Total & & $\mathbf{6 5}$ \\
\hline Age (years) & Under 5 & 2 \\
\cline { 2 - 3 } & $5-15$ & 10 \\
\cline { 2 - 3 } & Adults $(>15)$ & 53 \\
\hline Gender & Male & 48 \\
\cline { 2 - 3 } & Female & 17 \\
\hline History of fever $(\%)$ & & 100 \\
\hline Axillary temperature $\geq 37.5^{\circ} \mathrm{C}$ & $73.8(48 / 65)$ \\
\hline Geometric mean parasite density $(/ \mu l)$ & 69,535 \\
\hline
\end{tabular}

Thailand-Cambodia border areas, but the status of artemisinin in China was unclear. The spread of artemisinin resistance from Cambodia to China or the de novo occurrence of artemisinin resistance in China is a serious concern. This study was designed to evaluate clinical outcome after seven-day artesunate monotherapy for uncomplicated $P$. falciparum malaria in Yingjiang County along the China-Myanmar border and investigate the prevalence of anti-malarial drug resistance markers. A parallel study evaluating DHA-PIP was conducted simultaneously in another county, and the results of that study will be published elsewhere.

Yunnan province is located in southern China, with a population of approximately 30 million people in 129

Table 2 The allele frequency of the pre- and posttreatment of the two "treatment failure" patients

\begin{tabular}{|c|c|c|c|c|c|c|}
\hline \multirow[t]{3}{*}{ Sample } & \multicolumn{6}{|c|}{ PCR Product Size(s) (bp) } \\
\hline & \multicolumn{2}{|l|}{$m s p 2$} & \multirow[t]{2}{*}{ Glurp } & \multicolumn{3}{|l|}{ msp1 } \\
\hline & FC27 & $3 D 7 / 1 C$ & & RO33 & K1 & MAD20 \\
\hline P1-D0 & & 500 & 760 & 160 & & \\
\hline P1-D14 & 320 & 500 & 960 & & & 220 \\
\hline P2-D0 & 400 & & 600 & & & 160 \\
\hline P2-D14 & 350 & & 820 & & & 220 \\
\hline
\end{tabular}




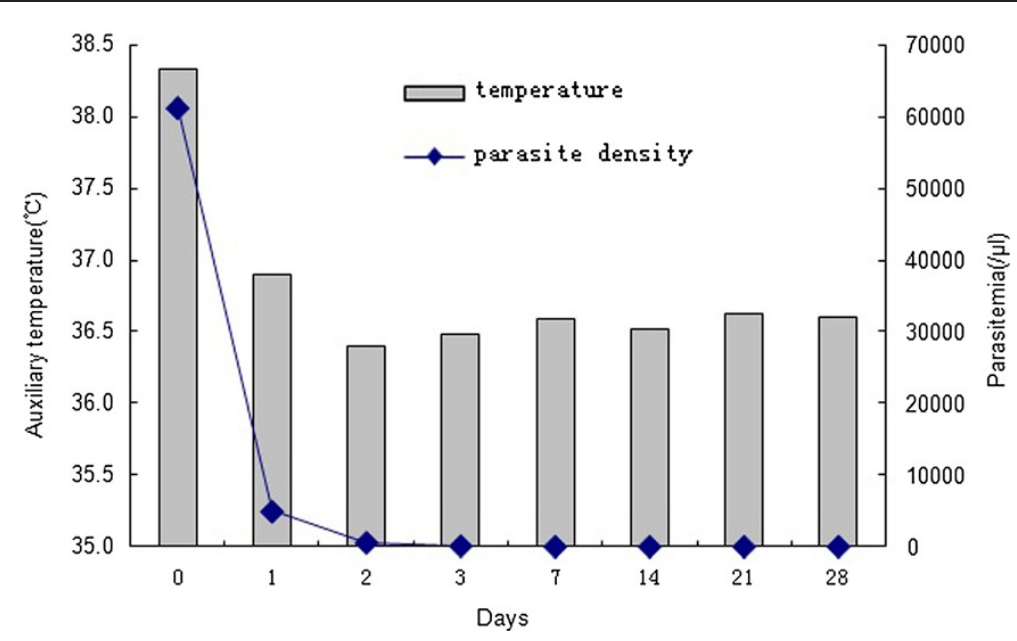

Figure 3 Average patient body temperature and parasite density on days $0,1,2,3,7,14,21$ and 28 after artesunate treatment for seven days.

counties. The province borders Myanmar, the Lao People's Democratic Republic and Vietnam. The border with Myanmar is $1,997 \mathrm{~km}$ long (as shown in Figure 1). Yingjiang County belongs to Dehong Prefecture with the total population of 288,691 people, which has the highest malaria incidence rate in China. The incidence in 2008 was 19.1 cases per $10,000,38 \%$ of which were P. falciparum.

The majority of patients included in the study were from Myanmar or had been infected in a region of Myanmar close to the Chinese border. Among the 65 patients with $P$. falciparum, two had early treatment failure (4.1\%), ie, confirmed re-infection. Therefore, this patient excluded as treatment failure with PCR correction until day 14. However, a key observation was the high rate of parasite positivity on day 3. A total of $18.5 \%$ of the enrolled patients were parasite positive on day 3 . The initial parasite density was high, which might partially explain the high day 3 positivity rate, but the response still suggested a lowered susceptibility to artesunate, which was lower than the response rate observed in western Cambodia in recent years. It is impossible to say whether these findings reflect a spread from Cambodia or local factors. Local factors include long-term access to artemisinin (since at least the early or mid1990s) and highly irregular treatment schedules in an area with continued moderate levels of transmission, as suggested by the predominance of malaria in young adults. Therefore, therapeutic efficacy tests should be continued, and the containment of artemisinin resistance in these areas should be prioritized.

Pfcrt and pfmdr1 genes have been proposed as molecular markers of chloroquine resistance, and they also influence $P$. falciparum susceptibility to mefloquine, quinine, halofantrine and artemisinin [21-23]. According

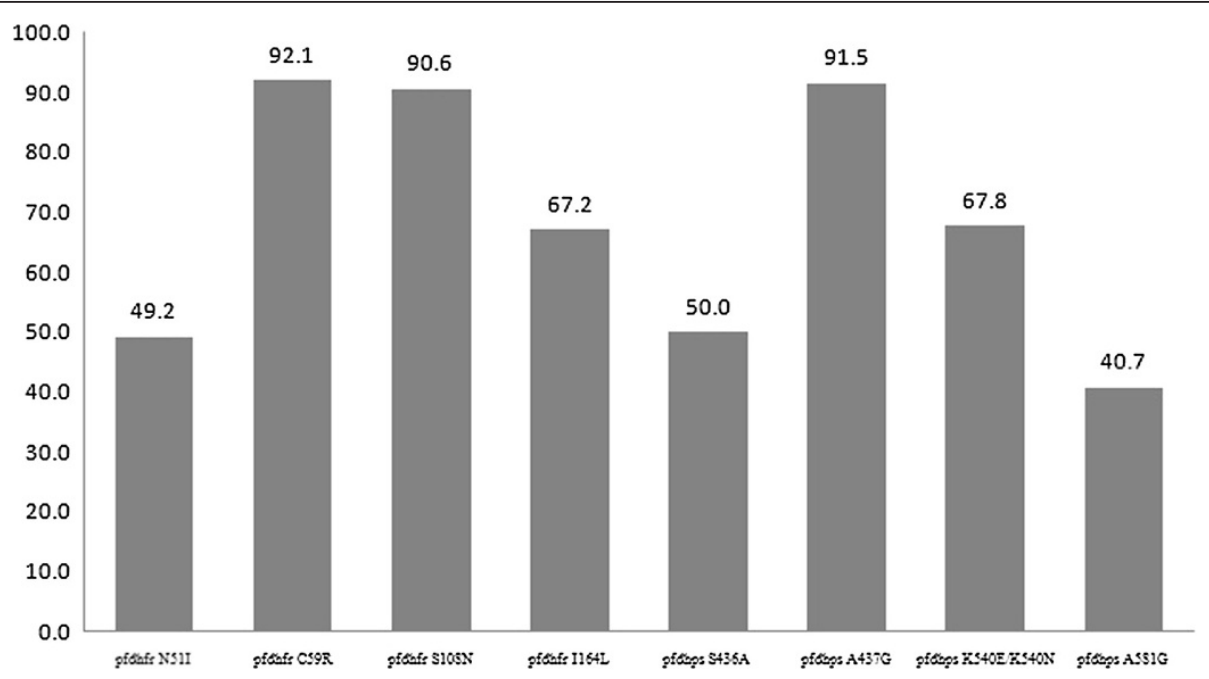

Figure 4 Frequency of pfdhfr and pfdhps haplotypes in samples from Yingjiang County. 
Table 3 Prevalence of pfdhfr (codons 51, 59, 108 and 164) and pfdhps (codons 436, 437, 540 and 581) mutants

\begin{tabular}{llcc}
\hline Gene & \multicolumn{1}{c}{ Mutation } & Number & Frequency (\%) \\
\hline pfdhfr & Quadruple mutants & $22(58)$ & 38.0 \\
\cline { 2 - 4 } & Triple mutants 51/59/108 & $9(58)$ & 15.5 \\
\cline { 2 - 4 } & Triple mutants 59/108/164 & $21(58)$ & 36.2 \\
\cline { 2 - 4 } & Double mutants 59/108 & $6(58)$ & 10.3 \\
\hline \multirow{2}{*}{ pfdhps } & Quadruple mutants & $13(55)$ & 23.6 \\
\cline { 2 - 4 } & Triple mutants 436/437/581 & $8(55)$ & 14.5 \\
\cline { 2 - 4 } & Double mutants 436/437 & $2(55)$ & 3.6 \\
\cline { 2 - 4 } & Double mutants 436/540 & $1(55)$ & 1.8 \\
\cline { 2 - 4 } & Double mutants 437/540 & $28(55)$ & 50.9 \\
\cline { 2 - 4 } & Double mutants 437/581 & $3(55)$ & 5.5 \\
\hline
\end{tabular}

to the results of molecular marker tests, there was a high prevalence of mutation in codon 76 of pfcrt. As might be expected from the previous studies on in vivo and in vitro chloroquine responses in China [24], the isolates of $P$. falciparum examined in the present study frequently contained the pfcrt K76T mutation, especially in Yunnan province. Although China has not used chloroquine to treat $P$. falciparum for over 30 years, the stable and high prevalence of this mutation may be caused by the continued use of chloroquine as a first-line drug for P. vivax over several decades. Compared with the high prevalence of $p f c r t$ mutations, there was no mutation in codons 86 or 1246 of $p f m d r 1$ in any sample. This result was consistent with other researchers' findings [25-27]. Until now, the relationship between the Y86 mutation and chloroquine resistance has not been confirmed, and there have been many conflicting results. The results in this research may indicate that $P$. falciparum in China is still sensitive to mefloquine, quinine, halofantrine and artemisinin.

The effects of $p f d h f r$ and $p f d h p s$ mutations on the mechanism of resistance to SP drugs have been well described. Pyrimethamine was used for the radical treatment of $P$. vivax in combination with primaquine 40 years ago [28]. Additionally, pyrimethamine was added to salt for prophylaxis in the 1980s [29]. Pyrimethamine plus primaquine has always been recommended as prophylaxis for specific populations in China [7] and sulphadoxinepyrimethamine were used in Myanmar in 1980's and the resistance at various levels were common throughout the country in the beginning of 1990's [30]. This study found that the prevalence of quadruple, triple and double mutants at the China-Myanmar border was high, which suggested that these parasites are still resistant to antifolate anti-malarial drugs in China, two decades after SP was abandoned as a first-line anti-malarial [31]. This research also confirmed that the pfdhps A437G mutation confers resistance to sulphadoxine and is often coupled with the K540E mutant allele.
The mechanism of action of artemisinin remains controversial. One of its proposed mechanisms involves an interaction with the sarcoplasmic reticulum $\mathrm{Ca}^{2+}$ ATPase6. The analysis of naturally occurring pfatp6 polymorphisms in field isolates suggested that a polymorphism at codon 769 may be associated with the reduced susceptibility of these isolates to artemether in vitro [17]. In line with other investigators, there was any polymorphism in codons 263 or 769 , which are described as the key amino acids in the interaction between pfatp6 and artemisinins [32]. However, two new mutations (N683K and R756K) were found in parasite isolates in this study, and the mutation of position 683 has not been reported previously [33]. The mutations observed in this study and in previous studies [34-36] could be indirectly implicated in this interaction if they are associated with artemisinin susceptibility. But one recent study [37] on emergence of artemisinin-resistant malaria on the western border of Thailand showed the proportion of variation in parasite clearance attributable to parasite genetics increased doubling compared with year 2001-2004 and year 2007-2010. Considering the development of artemisinin combination therapy and the possible implication of pfatp 6 in artemisinin resistance, all of the polymorphisms in this gene should be carefully monitored.

\section{Conclusion}

Slow parasite clearance and suspected artemisinin resistance was observed in Yingjiang County at the ChinaMyanmar border area, therefore it should be strengthen further on parasite clearance time or clearance half life to confirm the resistance status. And now Yunnan province of China, as part of Greater Mekong Sub-region, has already involved in "Greater Mekong Sub-region Programme for Artemisinin Resistance Containment", which will be launching this year or next year.

Also, this study provided basic data on artemisinin resistance in this area, which will be helpful for other countries in the Mekong sub-region. Moreover, molecular epidemiology should be continued as routine surveillance and to provide complementary information to assess the appropriateness of current policies based on artemisinin derivatives.

\section{Competing interests}

The authors hereby certify that no conflict of interest of any kind occurred in the framework of this study.

\section{Authors' contributions}

FH was responsible for the molecular genetic studies, participated in the field work, and drafted the manuscript. LT was responsible for the overall study design and was involved in all stages of the study, including field work. HY was involved in the design of the field work. SZ contributed to the molecular genetic studies and data analysis. XS and HL contributed to the field study, patient follow-up and data analysis. All of the authors read and approved the final manuscript. 


\section{Acknowledgements}

We thank two anonymous reviewers for their valuable comments and suggestions. This work was supported by the WHO Mekong Malaria Programme (WP/08/MVP/000512 and WP/10/MVP/005837) and a Project supported by the Chinese Centre for Disease Control and Prevention for Young Scientists of China (Grant No. 2011A105).

\section{Author details}

${ }^{1}$ National Institute of Parasitic Diseases, Chinese Centre for Disease Control and Prevention; WHO Collaborating Centre for Malaria, Schistosomiasis and Filariasis; Key Laboratory of Parasite and Vector Biology, Ministry of Health, Shanghai 200025, PR China. ${ }^{2}$ Yunnan Institute of Parasitic Diseases, Puer 665000, PR China.

Received: 8 May 2012 Accepted: 17 July 2012 Published: 16 August 2012

\section{References}

1. WHO: WHO Malaria report. Geneva: World Health Organization; 2009

2. Dondorp AM, Nosten F, Yi P, Das D, Phyo AP, Tarning J, Lwin KM, Ariey F, Hanpithakpong W, Lee SJ, Ringwald P, Silamut K, Imwong M, Chotivanich K, Lim P, Herdman T, An SS, Yeung S, Singhasivanon P, Day NP, Lindegardh N, Socheat $D$, White $\mathrm{NJ}$ : Artemisinin resistance in Plasmodium falciparum malaria. N Engl J Med 2009, 361:455-467.

3. WHO: Global report on antimalarial drug efficacy and drug resistance: 2000 2010. Geneva: World Health Organization; 2010.

4. WHO: World malaria report. Geneva: World Health Organization; 2010.

5. Zhou SS, Wang Y, Li Y: Malaria situation in the People's Republic of China in 2010. Chin J Parasitol Parasit Dis 2011, 29:401-403.

6. WHO: Guidelines for the treatment of malaria: World Health Organization; 2010.

7. Antimalarial drug policy in China. http://www.moh.gov.cn/publicfiles/ business/htmlfiles/mohjbyfkzj/s3594/200907/41610.htm.

8. Djimde A, Doumbo OK, Cortese JF, Kayentao K, Doumbo S, Diourte Y, Dicko A, Su XZ, Nomura T, Fidock DA, Wellems TE, Plowe CV, Coulibaly D: A molecular marker for chloroquine-resistant falciparum malaria. $N$ Engl J Med 2001, 344:257-263.

9. Ursing J, Kofoed PE, Rodrigues A, Rombo L, Gil JP: Plasmodium falciparum genotypes associated with chloroquine and amodiaquine resistance in Guinea-Bissau. Am J Trop Med Hyg 2007, 76:844-848.

10. Picot S, Olliaro P, de Monbrison F, Bienvenu AL, Price RN, Ringwald P: A systematic review and meta-analysis of evidence for correlation between molecular markers of parasite resistance and treatment outcome in falciparum malaria. Malar J 2009, 8:89.

11. Babiker HA, Pringle SJ, Abdel-Muhsin A, Mackinnon M, Hunt P, Walliker D: High-level chloroquine resistance in Sudanese isolates of Plasmodium falciparum is associated with mutations in the chloroquine resistance transporter gene pfcrt and the multidrug resistance Gene pfmdr1. $J$ Infect Dis 2001, 183:1535-1538.

12. Basco LK, Ringwald P: Molecular epidemiology of malaria in Yaounde, Cameroon. VI. Sequence variations in the Plasmodium falciparum dihydrofolate reductase-thymidylate synthase gene and in vitro resistance to pyrimethamine and cycloguanil. Am J Trop Med Hyg 2000, 62:271-276.

13. Hyde JE: Drug-resistant malaria - an insight. FEBS J 2007, 274:4688-4698.

14. Kublin JG, Dzinjalamala FK, Kamwendo DD, Malkin EM, Cortese JF, Martino LM, Mukadam RA, Rogerson SJ, Lescano AG, Molyneux ME, Winstanley PA, Chimpeni P, Taylor TE, Plowe CV: MolecnstitutionalAuthorNameular markers for failure of sulfadoxine-pyrimethamine and chlorproguanildapsone treatment of Plasmodium falciparum malaria. J Infect Dis 2002, 185:380-388.

15. Lynch C, Pearce R, Pota H, Cox J, Abeku TA, Rwakimari J, Naidoo I, Tibenderana J, Roper C: Emergence of a dhfr mutation conferring highlevel drug resistance in Plasmodium falciparum populations from southwest Uganda. J Infect Dis 2008, 197:1598-1604.

16. Imwong M, Pukrittayakamee S, Pongtavornpinyo W, Nakeesathit S, Nair S, Newton P, Nosten F, Anderson TJ, Dondorp A, Day NP, White NJ: Gene amplification of the multidrug resistance 1 gene of Plasmodium vivax isolates from Thailand, Laos, and Myanmar. Antimicrob Agents Chemother 2008, 52:2657-2659.
17. Uhlemann AC, Cameron A, Eckstein-Ludwig U, Fischbarg J, Iserovich $P$, Zuniga FA, East M, Lee A, Brady L, Haynes RK, Krishna S: A single amino acid residue can determine the sensitivity of SERCAs to artemisinins. Nat Struct Mol Biol 2005, 12(7):628-629.

18. WHO: Methods and techniques for clinical trials on antimalarial drug efficacy: genotyping to identify parasite populations. Geneva: World Health Organization; 2008. http://www.who.int/malaria/resistance.

19. Australian New Zealand Clinical Trials Registry. https://www.anzctr.org.au.

20. Pearce RJ, Drakeley C, Chandramohan D, Mosha F, Roper C: Molecular determination of point mutation haplotypes in the dihydrofolate reductase and dihydropteroate synthase of Plasmodium falciparum in three districts of northern Tanzania. Antimicrob Agents Chemother 2003, 47:1347-1354

21. Lin JT, Juliano JJ, Wongsrichanalai C: Drug-resistance malaria: the era of ACT. Curr Infect Dis Rep 2010, 12:165-173.

22. Sidhu $A B$, Verdier-Pinard D, Fidock DA: Chloroquine resistance in Plasomodium falciparum malaria parasites conferred by pfcrt mutations. Science 2002, 298:210-213.

23. Duraisingh MT, Jones $P$, Sambou I, von Seidlein L, Pinder M, Warhurst DC: The tyrosine- 86 allele of the pfmdr1 gene of Plasmodium falciparum is associated with increased sensitivity to the anti-malarials mefloquine and artemisinin. Mol Biochem Parasitol 2000, 108:13-23.

24. Liu DQ, Liu RJ, Ren DX, Gao DQ, Zhang CY, Qiu CP, Cai XZ, Ling CF, Liang AH, Tang Y: Alteration in resistance of Plasmodium falciparum to chloroquine after cessation of chloroquine medication for twelve years. Zhongguo $\mathrm{Ji}$ Sheng Chong Xue Yu Ji Sheng Chong Bing Za Zhi 1992, 10:241-244.

25. Guan YY, Tang LH, Hu L, Feng XP, Liu DQ: The point mutations in Pfert and Pfmdr1 genes in Plasmodium falciparum isolated from Hainan Province. Chin J Parasitol Parasit Dis 2005, 23:135-139.

26. Yang Z, Zhang Z, Sun X, Wan W, Cui L: Molecular analysis of chloroquine resistance in Plasmodium falciparum in Yunnan Province China. Trop Med Int Health 2007, 12:1051-1060.

27. Zhang GQ, Guan YY, Zheng B, Wu S, Tang LH: Molecular assessment of Plasmodium falciparum resistance to antimalarial drugs in China. Trop Med Int Health 2009, 14:1266-1271.

28. Zhang SX: Efficiency of Pyrimethamine combined with primaquine for radical treatment of P.vivax. Xinyang Medical and Health compilation 1965, 2:32. unpublished data.

29. Wang RZ: Efficiency of pyrimethamine salt for P.vivax prophylaxis. Railway Medicine 1981, 4:246-247.

30. Ejov MN, Tun T, Aung S, Sein K: Response of falciparum malaria to different antimalarials in Myanmar. Bull World Health Organ 1999, 77:244-249.

31. Triglia T, Menting JG, Wilson C, Cowman AF: Mutations in dihydropteroate synthase are responsible for sulfone and sulfonamide resistance in Plasmodium falciparum. Proc Natl Acad Sci 1997, 94:13944-13949.

32. Jambou R, Legrand E, Niang M, Khim N, Lim P, Volney B, Ekala MT, Bouchier C, Esterre P, Fandeur T, Mercereau-Puijalon O: Resistance of Plasmodium falciparum field isolates to in-vitro artemether and point mutations of the SERCA-type PfATPase6. Lancet 2005, 366:1960-1963.

33. Bertaux L, le Quang $H$, Sinou V, Thanh NX, Parzy D: New PfATP6 mutations found in Plasmodium falciparum isolates from Vietnam. Antimicrob Agents Chemother 2009, 53:4570-4571.

34. Dahlström S, Veiga MI, Ferreira P, Mårtensson A, Kaneko A, Andersson B, Björkman A, Gil JP: Diversity of the sarco/endoplasmic reticulum Ca2 +-ATPase orthologue of Plasmodium falciparum (PfATP6). Infect Genet Evol 2008, 8:340-345.

35. Ibrahim ML, Khim N, Adam HH, Ariey F, Duchemin JB: Polymorphism of PfATPase in Niger: detection of three new point mutations. Malar J 2009, 8:28.

36. Menegon M, Sannella AR, Majori G, Severini C: Detection of novel point mutations in the Plasmodium falciparum ATPase6 candidate gene for resistance to artemisinins. Parasitol Int 2008, 57:233-235.

37. Phyo AP, Nkhoma S, Stepniewska K, Ashley EA, Nair S, McGready R, Ler Moo C, Al-Saai S, Dondorp AM, Lwin KM, Singhasivanon P, Day NP, White NJ, Anderson TJ, Nosten F: Emergence of artemisinin-resistant malaria on the western border of Thailand: a longitudinal study. Lancet 2012, 379:1960-1966.

doi:10.1186/1475-2875-11-278

Cite this article as: Huang et al:: Therapeutic efficacy of artesunate in the treatment of uncomplicated Plasmodium falciparum malaria and anti-malarial, drug-resistance marker polymorphisms in populations near the China-Myanmar border. Malaria Journal 2012 11:278. 